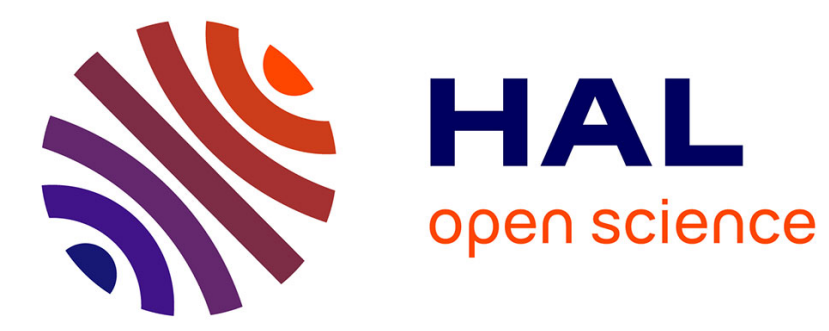

\title{
Strengthening and drying rate of a drying emulsion layer
}

Marie Goavec, Stéphane Rodts, Vincent Gaudefroy, Mathilde Coquil, Emmanuel Keita, Julie Goyon, Xavier Chateau, Philippe Coussot

\section{To cite this version:}

Marie Goavec, Stéphane Rodts, Vincent Gaudefroy, Mathilde Coquil, Emmanuel Keita, et al.. Strengthening and drying rate of a drying emulsion layer. Soft Matter, 2018, 14 (42), pp.8612-8626. 10.1039/C8SM01490F . hal-01980615

\section{HAL Id: hal-01980615 https://hal.science/hal-01980615}

Submitted on 19 Apr 2019

HAL is a multi-disciplinary open access archive for the deposit and dissemination of scientific research documents, whether they are published or not. The documents may come from teaching and research institutions in France or abroad, or from public or private research centers.
L'archive ouverte pluridisciplinaire HAL, est destinée au dépôt et à la diffusion de documents scientifiques de niveau recherche, publiés ou non, émanant des établissements d'enseignement et de recherche français ou étrangers, des laboratoires publics ou privés. 


\title{
Strengthening and drying rate of a drying emulsion layer
}

\author{
M. Goavec, S. Rodts, V. Gaudefroy", M. Coquil, E. Keita, J. Goyon, X. Chateau, P. Coussot \\ Université Paris-Est, Laboratoire Navier (ENPC-IFSTTAR-CNRS), 2 Allée Kepler, 77420 Champs sur \\ Marne, France \\ *IFSTTAR Nantes, Département MAST, Route de Bouaye, 44344 Bouguenais, France
}

\begin{abstract}
From direct observations and MRI measurements we demonstrate that during the drying of a direct (oil in water) emulsion the whole system essentially concentrates homogeneously, which leads to shrinkage, without air penetration. The structure and mechanical strength of this concentrated bulk are not significantly different from those of an emulsion directly prepared at this higher concentration. Despite this phenomenon, the drying rate continuously and rapidly decreases as the water content decreases, in contrast with the drying of a simple granular packing. This results from a concentration gradient which develops towards the free surface of the sample where the oil droplets finally coalesce, ultimately forming an oil layer covering the sample through which the water molecules have to diffuse before evaporating. Moreover, as during the process, the liquid is transported towards the free surface where it evaporates, surfactants accumulate and tend to form a thin solid layer below the oil layer, which tends to further reduce the drying rate.
\end{abstract}

\section{Introduction}

Direct (oil-in-water) emulsions are used in various fields, in particular for their ability to dry and ultimately form uniform liquid or solid surfaces: cosmetics [1], [2], paints [3], [4] , paving industry [5], foodstuffs (in particular spray-drying) [6], [7]. In these applications, it is essential to know how the layer dries, i.e. how and at what velocity it strengthens. The knowledge available in that field is still somewhat limited.

Emulsion drying means that the continuous phase, i.e. the aqueous solution, evaporates. Consequently, the dispersed phase concentrates, i.e. the oil droplets approach each other at a closer (average) distance. When the droplet concentration is larger than a critical value the droplets form a (soft) solid structure [8], [9]. We are then dealing with a solid porous media (the droplet network) from which a liquid has to be extracted.

The mechanisms of drying of non-deformable, homogeneous porous structures initially filled with a liquid (perfectly wetting the solid) and submitted to a constant air flux along one of its external surface usually follows a well admitted scenario [10]-[17]. The drying rate remains constant as the sample significantly desaturates, despite air entrance in the medium: this is the CRP (Constant Rate Period), which results from two effects. (i) Any withdrawing of liquid leads to a homogeneous redistribution of the liquid throughout the sample due to capillary effects, which tend to make uniform the Laplace pressure at any point in the liquid network. (ii) This maintains a large density of small liquid patches around the sample free surface, which keeps approximately constant the boundary conditions in terms of vapor field in this region, which in turn induces a constant drying 
rate. Thus, in this regime, the drying rate is mainly imposed by external conditions. When the liquid film size is too thin or, equivalently, the external evaporation rate is too large, capillary effects are unable to re-equilibrate sufficiently rapidly the liquid network, which tends to induce a complete drying of some region situated below the free surface. As the thickness of this region grows, the rate of drying decreases because now, before reaching the external air flux, the water vapor molecules have to diffuse through the dry region. This is the Falling Rate Period (FRP). During this period, the drying rate becomes much less dependent on the external air flux. Such a scheme remains qualitatively valid down to nanometric pore size [18].

With emulsions, there are first two major complications concerning this scheme. The elements composing the structure, i.e. the droplets, can deform when submitted to some stress, so that we are dealing with a soft solid, which may deform under capillary stresses; if they are too close to each other they can coalesce, which leads to the gradual formation of a continuous oil phase [19]. An additional complication comes from the fact that the droplet concentration increases and the resulting droplet deformation or coalescence, do not necessarily occur homogeneously throughout the sample. Indeed, evaporation generally takes place along some interface of the emulsion with air, often situated around a material boundary. This implies that water will have to move more or less rapidly towards this interface to eventually evaporate. Depending on the speed of this displacement, the droplet concentration can vary from uniform to strongly heterogeneous (with a peak at the approach of the interface). This specific distribution will affect the rate of drying and the coalescence, which will in turn affect the rate of drying and the next evolution of the distribution. A situation with some analogy was observed with a microgel suspension distributed in a porous medium for which a strong gradient of microgel concentration was observed [20]. Finally we can expect that the evolution of the drying characteristics of emulsion depend on a complex coupling of these different physical effects [4].

Less information is available concerning emulsion drying than for solid porous materials. With the help of MRI (Magnetic Resonance Imaging) it was shown that in a film of the order of $200 \mu \mathrm{m}$ thick the concentration distribution remains uniform during drying for low air flux velocity, but exhibits a growing gradient as the air flux increases [21]. With a much thicker layer of silicone emulsion some gradients of concentration were observed during the drying process, along with a continuous decrease of the drying rate [22]. The major result was nevertheless the identification of several effects occurring around the upper interface (in contact with air), such as a segregation of surfactants and the formation of an oil layer of growing thickness resulting from the coalescence of droplets [22]-[24].

Other works provided insight on the drying mechanisms for emulsion samples under different conditions. With a 2D emulsion in a capillary it was shown that during the drying process, the droplets at the front of the emulsion (i.e. along the evaporation region) finally coalesce either from the front or in the bulk depending on the surfactant concentration [25]. This effect was then explained with a model predicting the evolution of the gradient of droplet concentration in time [26]. For an emulsion layer enclosed between two solid plates at first a CRP was observed, followed by a FRP which was explained by the further approach and deformation of the droplets reducing the liquid path [27]. Other works focused on the drying of a drop of emulsion, which leads to phenomena that are more complex since evaporation now occurs from a very large interface of the sample and at different rates, inducing heterogeneities along the various directions. Internal observations of the evolution of concentration inside a drop of emulsion during its drying was allowed by NMR [28]-[30], 
but optical combined with scanning electron microscopy allowed to present a further scheme of the effects occurring in such a case coalescence, cracking, crack healing, etc [31].

Our work aims at determining more precisely the drying behavior of a layer of emulsion, in order to understand the main physical effects governing the strengthening and the drying rate, and the possible coupling between these two aspects. In that aim we follow the rheological characteristics of the emulsion during drying and the detailed spatial distribution of the two phases of the system in time. Note that we use a relatively thick layer of emulsion, namely larger than one centimeter, as compared to usual applications in cosmetics or civil engineering (paints) which involve submillimetric layers. The interest is that the larger scale allows for better relative resolution in internal imaging, while the physical phenomena behind the process are similar as long as the continuum assumption is valid, i.e. as long as the sample thickness is much larger than the size of the elementary components of the emulsion (typically a few microns). Then, these physical effects may lead to different evolutions of the sample depending on the boundary conditions (which include the sample thickness). Thus, this approach will allow to identify physical processes and provide data to be used for model validation. Our results in particular show that even a thick layer of emulsion essentially concentrates homogeneously, which leads to shrinkage, without air penetration. The structure and mechanical strength of this concentrated bulk is not significantly different from that of an emulsion directly prepared at this higher concentration. Despite this phenomenon, the drying rate continuously and rapidly decreases as the water content decreases, which shows that the evolutions of the drying rate and the emulsion strength are not directly coupled. It is finally shown that we can expect similar behavior for thinner layers.

\section{Materials and methods}

\section{Materials}

We prepared an oil-in-water emulsion using silicone oil with a viscosity of $20 \mathrm{mPa}$.s (Chimie Plus). The silicone oil droplets are stabilized by a surfactant, TTAB (Alfa Aesar). The surfactant used is a quaternary ammonium salt with a critical micellar concentration (CMC) of $3.5 \mathrm{mM}$ and a solubility in water of $10 \%(\mathrm{w} / \mathrm{v})$, which are respectively equivalent to mass fractions of $0.12 \%_{\mathrm{wt}}$ and $9.09 \%_{\mathrm{wt}}$. The emulsion was prepared using a Silverson L4RT rotor-stator mixer. The oil was progressively added to an aqueous surfactant solution ( $\left.3 \%_{\mathrm{wt}}\right)$ at $500 \mathrm{rpm}$. Once all the oil was added, the rotation speed was increased to 5000rpm in 500rpm steps. The emulsion obtained has an initial oil volume fraction of $81 \%$. For some experiments, the emulsion was diluted with distilled water to reach an oil volume fraction of $61 \%$. This emulsion was stable to sedimentation for a couple days after preparation (in absence of drying).

\section{Confocal microscopy}

The size of the emulsion oil droplets was estimated using confocal microscopy. To do so, fluorescein (Fluorescein sodium, Fluka Chemica) was added to the aqueous continuous phase and a drop of emulsion was deposited on a glass slide and observed with a confocal microscope (Carl Zeiss LSM 700) equipped with a $488 \mathrm{~nm}$ laser. These measurements allowed us to monitor the emulsion stability. Indeed after preparation, the average size of emulsion droplets was found to be about 2-5 $\mu \mathrm{m}$. During storage of the emulsion in sealed containers at ambient temperature, the mean droplet 
diameter did not evolve significantly over time. These observations also allowed us to monitor the droplet size evolution during drying (see Macroscopic drying measurements). The samples taken from the drying emulsion were strongly diluted with fluorescent water (+ TTAB) and placed on a glass slide. Several images of the emulsion were taken in order to have a large sampling of emulsion droplets (more than a thousand droplets). The radius of the droplets was then measured using Image J and Matlab software and used to establish the oil droplet size distribution in volume percentage:

$$
\%_{V}(i)=\frac{n_{i} 4 / 3 \pi r_{i}^{3}}{\sum_{i} n_{i} 4 / 3 \pi r_{i}^{3}}
$$

Where $n_{i}$ is the number of droplets with a radius of $r_{i}$.

\section{Drying experiments}

Drying tests were performed on emulsion samples contained in glass Petri dishes. The dishes were coated beforehand with a thin layer of perfluorinated grease (Krytox GPL 205, DuPont). The surface was thus non-adhering, allowing the sample to slide along the walls and bottom of the Petri dish. The sample diameter was either $5.6 \mathrm{~cm}$ or $9 \mathrm{~cm}$ (for the MRI tests). The material remained in contact with the walls, i.e. we did not observe any radial contraction during the experiments. We deduce that drying essentially occurs along the vertical sample axis, leading to a one-dimensional description of the phenomenon. Therefore, the sample diameter has a priori no impact on our results. The initial sample thickness was about $1.5 \mathrm{~cm}$.

\section{Macroscopic drying measurements}

Simple drying tests were carried out using a dry airflow blowing along the top surface of the emulsion sample (see Figure 1) at a temperature of $21 \pm 1^{\circ} \mathrm{C}$. The airflow was directed using a channel with a section of $60 * 4 \mathrm{~mm}^{2}$. A circular opening at the end of the channel $(\varnothing=6 \mathrm{~cm})$ allowed us to insert the sample and align it with the lower channel wall. The airflow rate was regulated using a manometer placed upstream of the channel and its velocity measured with a flowmeter at the end of the drying channel. The sample was placed on a scale, connected to a computer. This allowed us to record the mass of the emulsion sample $M_{E}$ every five minutes. Since the silicone oil negligibly evaporates during our tests (we did not observe any mass change of an oil layer exposed to air flux over 100 hours), the oil mass in the emulsion is equal to the initial mass of oil in the emulsion written $M_{O}$, and the mass loss $\Delta M(t)$ observed during drying corresponds to the mass of evaporated water. Therefore, at all times the volume of water in the sample is $M_{W}(t)=M_{W_{0}}-\Delta M(t)$ with $M_{W_{0}}$ the initial mass of water in the emulsion.

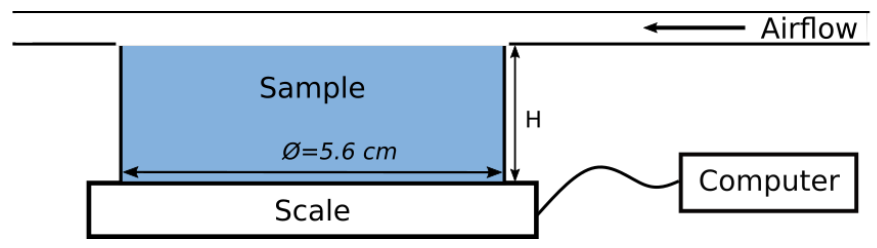

Figure 1 : Experimental setup for the macroscopic measurements of drying of emulsions.

From these measurements, we can compute the oil volume fraction $\phi(t)$ as a function of time: 


$$
\phi(t)=\frac{\Omega_{o}}{\Omega_{o}+\Omega_{W}}
$$

in which $\Omega_{W}=M_{W} / \rho_{W}$ and $\Omega_{o}=M_{o} / \rho_{o}$ are the current water and oil volumes respectively, $\rho_{W}$ and $\rho_{O}$ being the water and oil density. Note that this definition implicitly assumes that there is just oil and water in the emulsion volume and no air, a point that will be discussed later in the paper. We can also compute the drying rate $V$ of the emulsion, defined as:

$$
V=-\frac{1}{\rho_{W} S} \frac{d M_{W}}{d t}
$$

with $S$ the sample surface area.

For some experiments, drying was also monitored through confocal microscopy in order to observe possible evolutions of the structure or the droplet size. To do so an emulsion containing fluorescein in the aqueous phase was put to dry with the setup described above. Samples were collected at regular time intervals and obtained by first removing the uppermost part of the drying emulsion ( $\approx 1 \mathrm{~mm}$ thick) with a thin spatula, allowing us to separate any supernatant oil and white crust from the rest of the drying emulsion. A sample was then taken with a spatula over the whole height of the sample and overall about $0.1 \mathrm{~g}$ of emulsion was collected. Part of the sample was directly placed on a glass slide to observe the droplet arrangement with the confocal microscope while the remaining sample was diluted with fluorescent water (+ TTAB) before being observed in order to estimate droplet size distribution (see Confocal microscopy). Note that this dilution could not affect the droplet size. The mass of the sample was recorded just before and after sampling in order to take into account the mass loss by sampling and adjust the corresponding drying curve.

\section{MRI measurements}

The drying experiments with in situ monitoring of the local oil and water content were performed in a vertical Magnetic Resonance Imaging (MRI) spectrometer (Bruker DBX 24/80) operating at 0.5T (20MHz proton frequency) with a measuring area $20 \mathrm{~cm}$ wide. A MRI compliant drying setup allowed to dry the sample directly in the MRI magnet during measurements. It consisted of a PMMA sample holder and a long vertical column through which a dry airflow was blown towards the top surface of the emulsion at a controlled rate. All experiments were carried out at room temperature $\left(T \approx 22^{\circ} \mathrm{C}\right)$.

Proton MRI detects hydrogen atoms, therefore in the case of water / silicone oil emulsions, those of the water and the oil phase. A dedicated protocol is used to separate the two fluids, based on the differences between their NMR relaxation times [32]. Other strategies based on the difference of chemical shift were not suited, due to the too high level of susceptibility induced by field inhomogeneities in our working conditions. In order to get one dimensional profiles of the content of the different fluids along the vertical direction, different MRI sequences were tested, which gave similar results (see Appendix 1), proving the reliability of our data. However, a systematic difference was found of about $1 \mathrm{~g}$ between the water mass deduced from MRI profiles (by integration over the sample height) and the weighed mass. For a given sample this mass difference remained constant from the beginning to the end of the drying. In other words, not all the water can be observed by MRI measurements. 


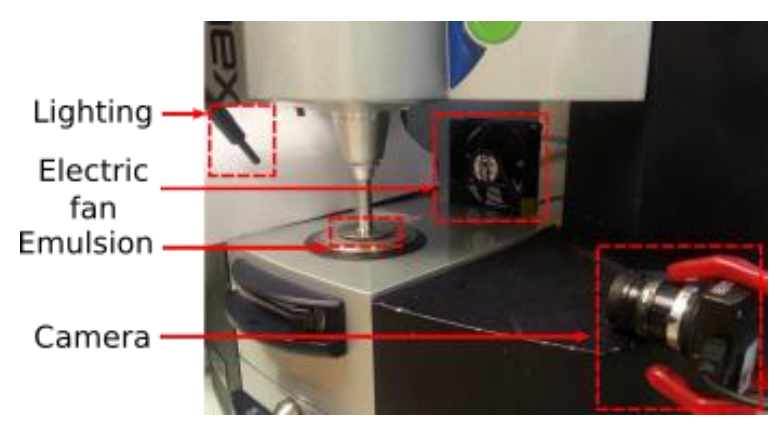

(a)

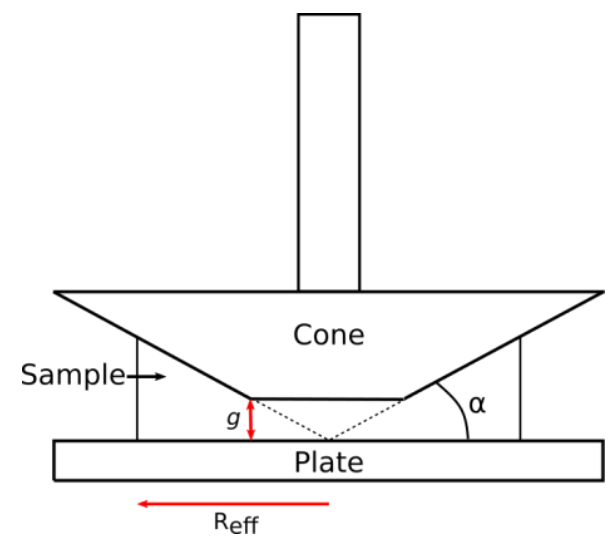

(b)

Figure 2 : (a) Experimental setup for the in-situ drying of an emulsion in the rheometer. The electric fan $(\varnothing=8 \mathrm{~cm})$ is placed at $13.5 \mathrm{~cm}$ from the center of the cone plate geometry. (b) Schematic representation of the sample held in the cone plate geometry during drying.

The evolution of drying emulsions was also monitored with a stress-controlled rheometer (Malvern Kinexus ultra+) equipped with a roughened cone and plate geometry $\left(2 \mathrm{~cm}\right.$ radius, $\alpha=4^{\circ}$ angle and gap at the truncature $g=0.15 \mathrm{~mm}$ ). The variations of the elastic and viscous moduli $G^{\prime}$ and $G^{\prime \prime}$ as a function of the strain amplitude (see Appendix 2) are typical of yield stress fluids [33]: a plateau of $G^{\prime}$ much higher than the $G^{\prime \prime}$ level is observed for small strain amplitude (solid regime), then a fast decrease of $G^{\prime}$ along with some increase of $G^{\prime \prime}$ beyond some critical deformation (liquid regime). For the drying tests, the sample was placed in the rheometer and the elastic and viscous moduli $G^{\prime}$ and $G^{\prime \prime}$ were measured at a fixed frequency of $1 \mathrm{~Hz}$ and shear strain of $1 \%$ at 20 min intervals after a $1 \mathrm{~min}$ pre-shear. Considering the behavior of these materials described above, for such a strain amplitude we a priori record the behavior of the emulsion in its solid regime. Simultaneously, the sample was dried in-situ using a small electric fan placed on one side of the rheometer (see Figure 2). The airflow it creates at the edge of the geometry has a speed of $1.2 \mathrm{~m} \cdot \mathrm{s}^{-1}$.

When the emulsions dried in the rheometer, they tended to shrink. This shrinkage induces a decrease of the effective sample radius $\left(R_{e f f}\right)$, which must be taken into account for the calculations of the effective values of the moduli, otherwise the standard rheometer calculations simply use the theoretical (initial) radius $R$. For that reason, the sample diameter was simultaneously monitored with a camera (Thorlabs DCC1545M) and recorded at 5 min intervals with ThorCam software. We then used ImageJ and Matlab software to measure the effective sample radius on the images and compute the effective elastic modulus $G_{e f f}^{\prime}=\left(R^{3} / R_{e f f}{ }^{3}\right) G_{a p p}^{\prime}$, in which $G_{a p p}^{\prime}$ is the elastic modulus value given by the rheometer (i.e. deduced from torque measurements assuming a constant sample radius $R$ ).

Moreover, as no oil evaporates during drying, the variation of the water volume $\Delta \Omega_{W}$ is equal to the emulsion volume variation $\Delta \Omega_{E}$ observed (still assuming no air in the emulsion), which we can compute: $\Delta \Omega_{W}=\Delta \Omega_{E}=\Omega_{E_{0}}-\left(2 \pi R_{e f f}{ }^{3} \tan \alpha / 3+\pi g^{3} / 3 \tan ^{2} \alpha\right)$ in which $\Omega_{E_{0}}$ is the initial emulsion volume. We can therefore compute the oil volume fraction $\phi$ as a function of time: $\phi(t)=\left(M_{o} / \rho_{O}\right) /\left(\Omega_{E_{0}}-\Delta \Omega_{W}\right)$. Finally we checked that the mass loss deduced from such 
measurements was in agreement with the mass variation found from direct weighing at the end of the test, which supports the assumption of no air entrance in the sample.

\section{Results}

\section{Rheology}

In order to look at the apparent behavior of the drying emulsion we followed the elastic and loss moduli of the emulsions during drying, starting from different initial concentrations. For an emulsion initially containing $61 \%$ oil, $G^{\prime}$ and $G^{\prime \prime}$ are initially relatively low (see Figure 3 ), in consistency with the fact that the emulsion looks liquid, even if it may already exhibit a small yield stress. As drying proceeds, $G$ first increases very sharply with the oil volume fraction. Once the volume fraction reaches $70 \%, G^{\prime}$ continues to increase but more progressively (in a logarithmic scale). Upon reaching $80 \%$ oil, the initial modulus has been multiplied by 100 . The viscous modulus is also initially very low, around a few Pascals, and then only slightly increases over the whole drying process (as compared to the elastic modulus), which means that the emulsion becomes an elastic solid as it concentrates during drying. The initial elastic moduli of emulsions with an initial volume fraction of 71 and $81 \%$ are much higher than for the emulsion initially at $61 \%$ (200 and 500 Pa respectively), and continue to increase throughout drying (see Figure 3). Interestingly enough, although the emulsions have different initial oil volume fractions and therefore elastic moduli, their elastic modulus evolves in the same manner and the curves superimpose quite well. It would therefore appear that the elastic moduli evolution follows a master curve.

On the other hand, the evolution of the viscous modulus differs from one emulsion to the other. On average, for the emulsions at different initial concentrations $G^{\prime \prime}$ increases continuously as the concentration increases, but this increase is faster for larger initial concentrations (see Figure 3).

These results are compared with measurements carried out on emulsions directly prepared at different oil volume fractions, obtained by diluting an initial $81 \%$ emulsion with continuous phase i.e. containing $3 \%_{\mathrm{wt}}$ surfactant (see Figure 3 ). We observe that the reference curve for the elastic moduli superimposes well with the emulsion drying curves.

On the other hand, the viscous modulus evolves towards larger values (by a factor 2 to 4 ) than that of the reference curve (see Figure 3). It may be suggested that, in contrast with the elastic modulus, the viscous modulus is affected by the surfactant concentration, which is not kept constant during drying. In order to test this, as first approximate, we can compute the apparent surfactant concentration in water $(\psi)$ as a function of the oil concentration: $\psi=\left(1-\phi_{0} / 1-\phi\right) \psi_{0}$, in which $\psi_{0}$ is the initial surfactant concentration and $\phi_{0}$ the initial oil fraction. It appears that the viscous modulus increases along a master curve when all data are now represented as a function of the factor of increase of $\psi$, which suggests that the surfactant concentration is effectively the main origin of these variations. However, we can hardly elaborate further about the exact physical process at the origin of this increase: the initial increase of $G^{\prime \prime}$ in proportion to the surfactant concentration does not correspond to what is expected for the viscosity of a suspension, which increases much more slowly with the concentration (typically as $1+2.5 \psi$ for $\psi$ less than a few percents) except at the approach of the "maximum packing fraction". In fact, a more precise estimation requires to take into account only the free surfactants molecules, i.e. not those adsorbed along the interface. However, this does not give mass concentration larger than $10 \%$ in all our tests, which is well below the critical concentration for micelle formation and thus cannot induce significant viscosity increase 
(see Appendix 3). Moreover, we have no explanation for the plateau in the second stage of evolution of $G^{\prime \prime}$ (see inset of Figure 3).

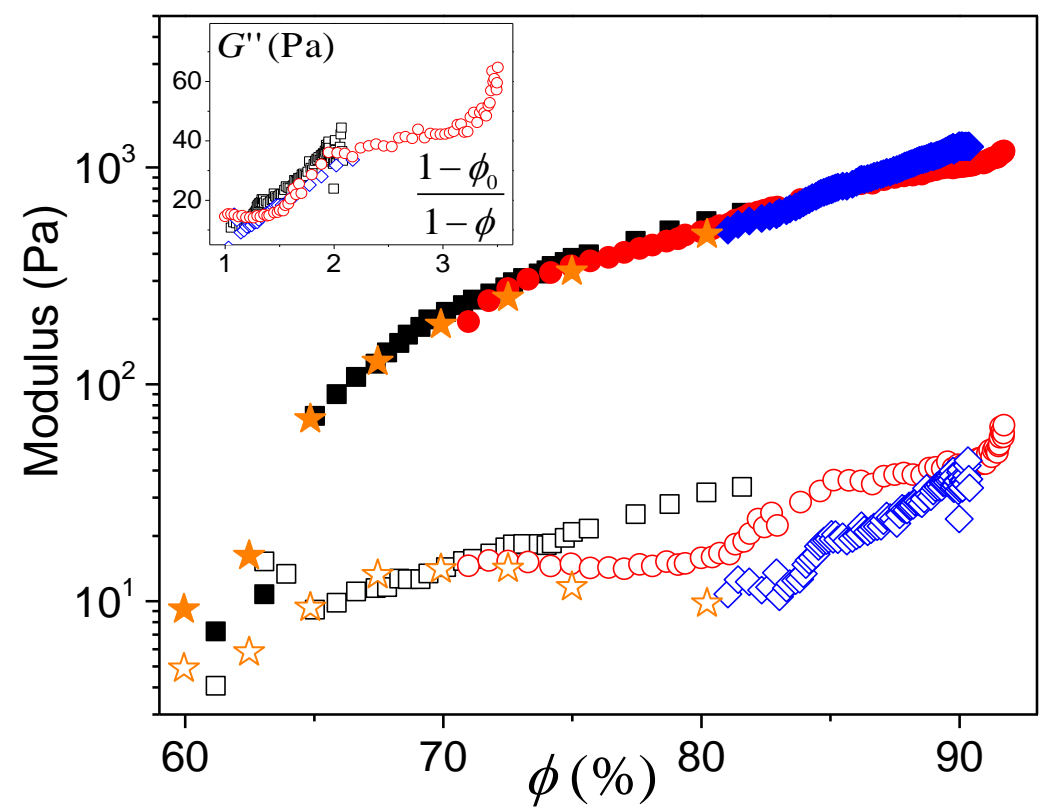

Figure 3 : Evolution of the elastic (filled symbols) and viscous (empty symbols) moduli as a function of oil volume fraction for $\mathrm{O} / \mathrm{W}$ emulsions with different initial oil volume fractions: $61 \%$ (black squares), $71 \%$ (red circles) and $81 \%$ (blue diamonds). All emulsions initially contain $\psi_{0}$ $=3 \%_{\mathrm{wt}}$ surfactant in the aqueous phase. The reference curves (orange stars) refer to the results obtained for an O/W emulsion at an initial oil volume fraction of $81 \%$ diluted to different oil volume fractions, while maintaining $3 \%_{\mathrm{wt}}$ surfactant in aqueous phase. The inset shows the viscous modulus as a function of the factor of increase of the surfactant concentration (see text).

The main point of these observations is that the elastic modulus, which essentially characterizes the rheological behavior of the emulsion since it is much larger than the viscous modulus, increases in the same way as that of a (homogeneous) emulsion directly prepared with the same water amount. We conclude that, from a mechanical point of view, all occurs as if, while drying, the emulsion homogeneously concentrated as a result of water extraction. This implicitly assumes that possible concentration gradients and air entrance have a negligible impact. We can have a look at these effects with MRI measurements.

\section{Local observations (MRI)}

The successive profiles giving the volume distribution of each component (oil and water) along the vertical axis during drying (see data in Figure 4 and Figure 5) provide detailed information on the process. First they allow to observe the continuous decrease of the heights of the water and oil profiles, which is indicative of a contraction of the emulsion: as water evaporates the volume of the emulsion decreases, and since no radial contraction was observed during drying, the sample free surface moves downwards. In addition, the profiles remain roughly horizontal, meaning that the water and oil concentration remain roughly uniform. We conclude that, as a first approximate, the emulsion essentially shrinks homogeneously. 
Moreover, we checked that no significant air entrance occurred during the process. In that aim we computed the apparent volume associated with the (total) mass of water and oil in each layer of the sample, as deduced from our MRI profile measurements. At any time during drying this volume appeared to be very close (within a few percents) to the theoretical one associated with a material layer occupying the whole available volume in the cylindrical container, meaning that a negligible air volume is contained in this layer.

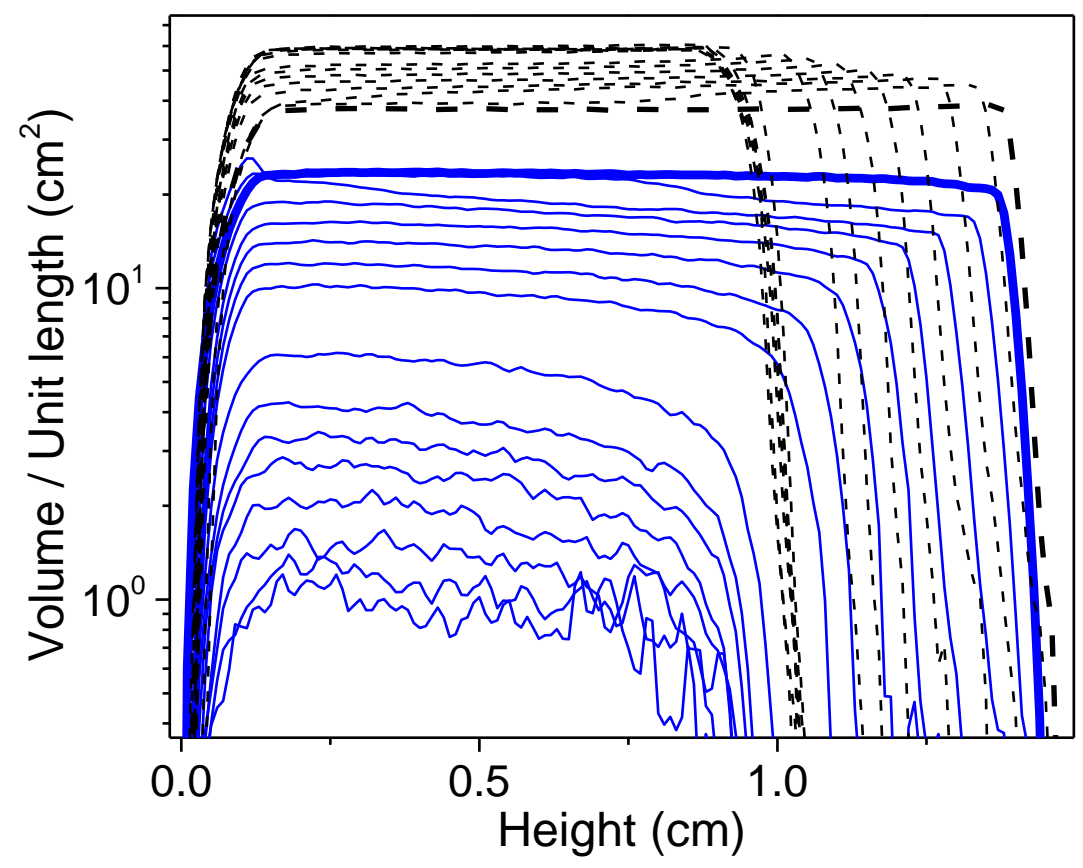

Figure 4 : Drying of an O/W emulsion initially composed of $61 \%$ silicone oil and containing $1.14 \%_{\mathrm{wt}}$ surfactant in aqueous phase $\left(\mathrm{H}=1.3 \mathrm{~cm}\right.$, air flow $\left.0.1 \mathrm{~m} . \mathrm{s}^{-1}\right)$. Water and oil distribution profiles are represented in solid (blue) and dashed (black) lines respectively. Initial profiles before drying has begun are represented in thick lines. Profiles are represented every $2 \mathrm{~h}$ after beginning of drying and every $11 \mathrm{~h}$ after $20 \mathrm{~h}$ of drying.

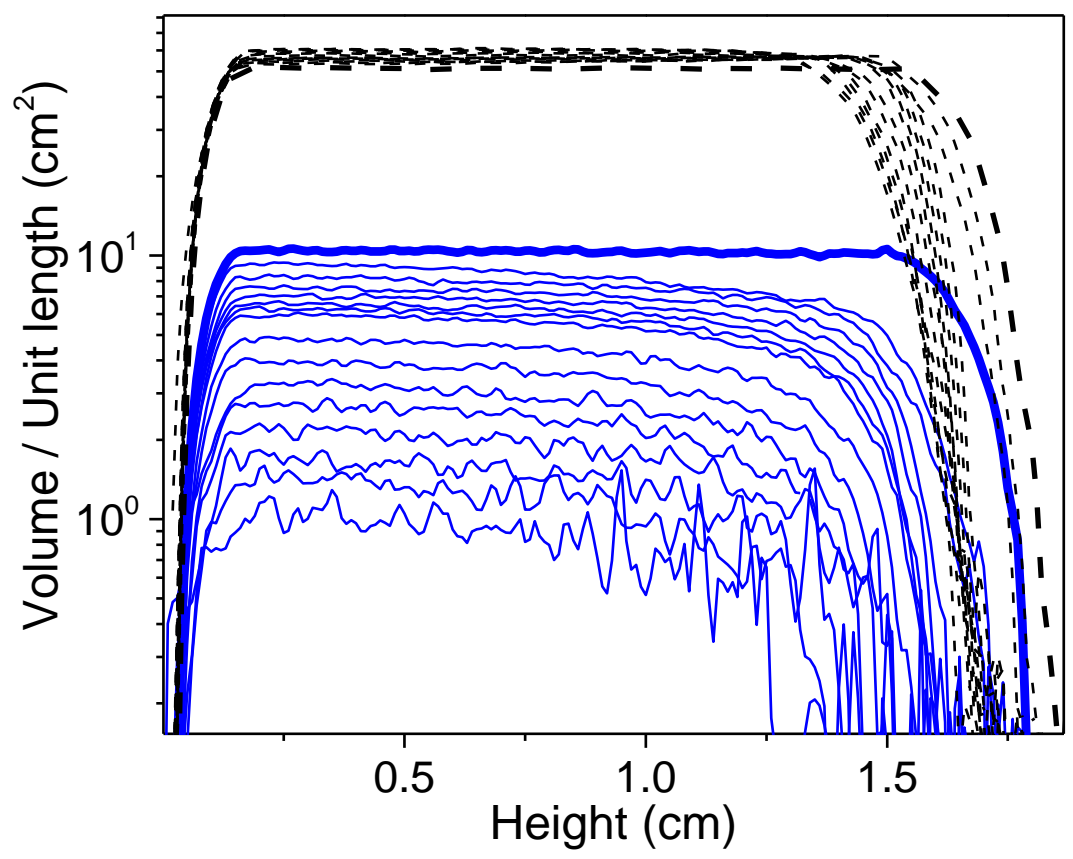


Figure 5 : Drying of an O/W emulsion initially composed of $81 \%$ silicone oil and containing $3 \%_{\text {wt }}$ surfactant in aqueous phase $\left(H=1.6 \mathrm{~cm}\right.$, air flow $\left.0.1 \mathrm{~m} . \mathrm{s}^{-1}\right)$. Water and oil distribution profiles are represented in solid (blue) and dashed (black) lines respectively. Initial profiles before drying has begun are represented in thick lines. Profiles are represented every $3 \mathrm{~h}$ after beginning of drying and every $10 \mathrm{~h}$ after $20 \mathrm{~h}$ of drying.

Looking now at the detailed evolution of the profile shape in time we can first remark that for a concentration of $61 \%$ during a first stage, some water near the surface is extracted first: the volume of water decreases in this area, while the bottom of the sample maintains the same water content (see Figure 4). Correspondingly, the oil volume increases near the surface. The water-depleted area, in the form of a step, spreads until it reaches the bottom of the sample, after 7 hours of drying which corresponds to an average residual water volume fraction of $31 \%$. Then the water content decreases in a more homogeneous manner, with nevertheless some gradient in water content at the approach of the free surface throughout drying. Correspondingly, the oil content increases approximately homogeneously, with some slight inverse gradient, which persists throughout drying.

For a higher initial oil volume fraction (e.g. 81\%), drying proceeds directly in the form of homogeneous shrinkage plus slight concentration gradient (see Figure 5). Incidentally, we can note that the profiles corresponding to similar total water content during drying of the emulsions with two different initial concentrations, appear similar (see Appendix 4). This tends to confirm that similar physical processes are at the origin of the contraction, which essentially depend on the current concentration and not on the history of drying.

To complete this description we can look at the impact of drying on the structure of the emulsion. In that aim we extracted small emulsion samples from the bulk at different times during drying, thus associated to different water contents, and we observed them through confocal microscopy. No particular evolution of the structure could be detected, and the droplet size distribution does not evolve up to a concentration of $93 \%$ (see Appendix 5).

All these observations show that, macroscopically, emulsion drying takes place essentially as a homogeneous shrinkage of the material, with a current structure similar to that of an emulsion directly prepared at the same concentration.

Under these conditions, the situation differs from the drying of a granular packing initially filled with water (see Introduction). The situation of emulsions rather resembles that of soft colloidal solids for which, as we withdraw the liquid from the matrix, the solid particles tend to approach further from each other, since creating some air-liquid interface between two such small solid elements would induce very large capillary effects that such a material could not resist. Thus, during a first period, a soft colloidal solid shrinks and the drying rate remains constant as liquid is still surrounding the solid phase [34]. In a second stage, the particles cannot approach closer anymore, so that the liquid has to find a way through the rigid network now formed; air penetrates the pores and the drying rate decreases [18], [34]. Finally, by analogy with these materials, we expect a constant drying rate for emulsions.

\section{Drying rate}

A typical evolution of the water content as a function of the rescaled time is presented in the inset of Figure 6, as well as the drying rate evolution (see Figure 6). Although fluctuations are observed a good reproducibility of the average evolution of the drying rate was found. From the water content 
vs time curve two regimes may be distinguished: in a first period the water content decreases linearly then, after a short transition regime, it decreases more slowly (see inset of Figure 6). These periods are better characterized from the drying rate curve (see Figure 6) where we can see that the drying rate remains high and almost constant as long as the oil fraction is smaller than about $70 \%$, then it starts to decrease rapidly. These results are unexpected with regards to our above reasoning, they mean that our assumption of approximately constant boundary conditions (i.e. wet free surface) is wrong. Something likely happens around the free surface, which was not detected in our first approach.

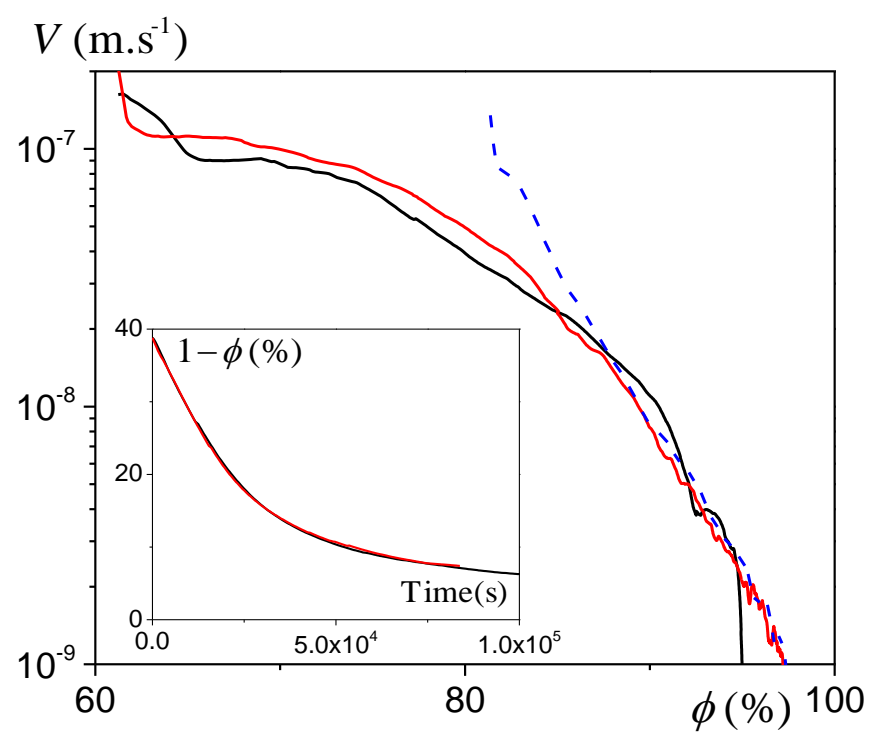

Figure 6 : Drying rate as a function of oil volume fraction for two identical O/W emulsions (continuous lines) initially composed of $61 \%$ silicone oil and containing $3 \%_{\mathrm{wt}}$ surfactant in aqueous phase ( $\mathrm{h}=6 \mathrm{~mm}$, air flow velocity $2.3 \mathrm{~m} . \mathrm{s}^{-1}$ ), and for an emulsion initially at $80 \%$ (dashed line). Inset shows water volume fraction as a function of rescaled time.

In order to understand this effect we can look at the sample aspect during drying. The emulsion is initially uniformly white and opaque (see Figure 7a and 7d). As drying proceeds, it gradually becomes transparent, starting from the surface (see Figure $7 \mathrm{~b}$ ) and spreading towards the bottom of the sample (see Figure 7c). We can also note in these images that the height of the emulsion surface decreases, however no radial contraction was observed. During drying, we observe that a solid white layer also forms progressively at the surface, as seen in Figures Figure $7 e$ and Figure $7 c$, although it is difficult to pinpoint the exact moment at which it begins to form: the white crust is difficult to observe as long as the emulsion retains its opacity.

We consider that the emulsion is dry once the general aspect of the emulsion (see Figure $7 \mathrm{c}$ and $7 \mathrm{f}$ ), and especially its composition, no longer evolves. The dried emulsions are essentially composed of a transparent phase, which occupies the bottom of the petri dish (see Figure 7c). Above this phase can be observed the thin white solid crust. Although the white layer is spread uniformly at the surface of the sample, it does not appear to have a uniform thickness, as some patches are very white and opaque while others are more transparent. We also observe a layer of supernatant transparent liquid as well, that floats above the thin white layer (see Figure $7 \mathrm{f}$ ). These observations are summarized in Figure 8. 


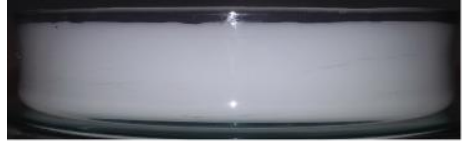

(a)

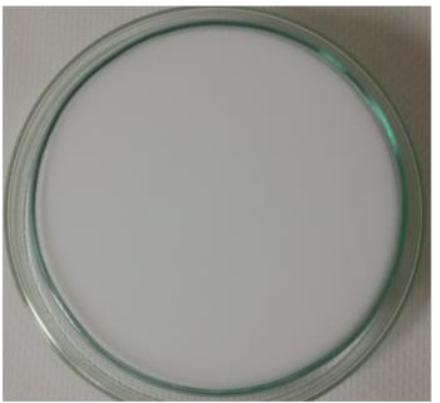

(d)

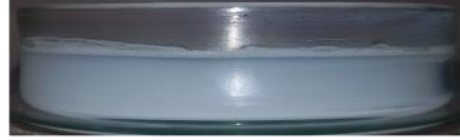

(b)

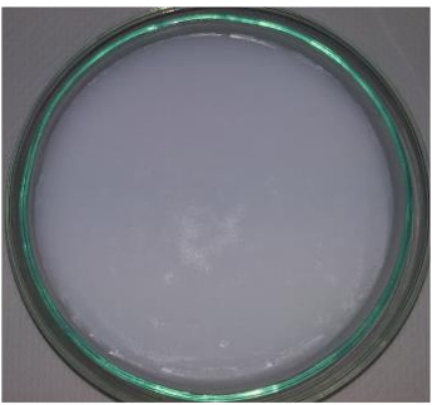

(e)

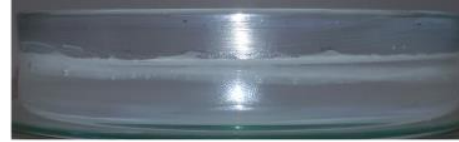

(c)

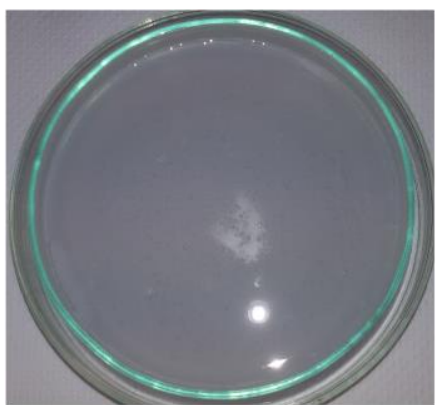

(f)

Figure 7 : Aspect of drying O/W silicone emulsion with $\phi(t=0)=61 \%$ at different stages. View from the side - (a) Initial state $\phi=61 \%$; (b) $\phi=97 \%$; (c) $\phi=98 \%$. View from above - (d) Initial state $\phi=61 \%$; (e) $\phi=85 \%$; (f) $\phi=98 \%$. The Petri dish has a height of $1.5 \mathrm{~cm}$ and a diameter of $9 \mathrm{~cm}$.

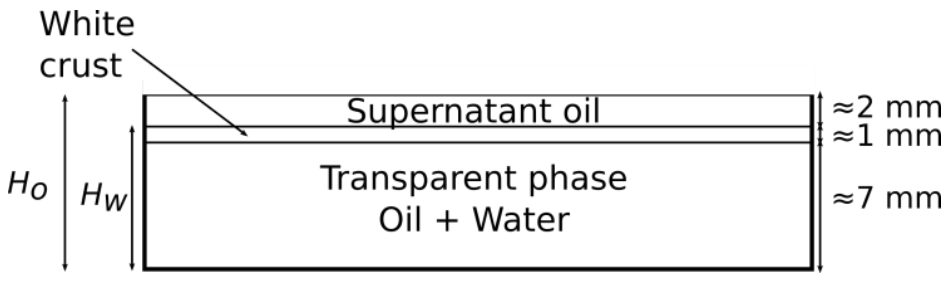

Figure 8 : Scheme of a dried O/W emulsion with $\phi(t=0)=61 \%$ and initial thickness of $1.5 \mathrm{~cm}$. The dried emulsion is composed of three layers: the transparent phase, the thin white crust and the supernatant oil. The thickness of each layer was measured using a ruler.

The supernatant liquid was sampled and observed to be non-miscible with water while its viscosity was measured by applying an increasing and decreasing stress ramp $\left(10^{-3}-1 \mathrm{~Pa}\right)$ and was found to be equal to $20 \mathrm{mPa} . \mathrm{s}$, which is the viscosity of the oil used to prepare the emulsion. Overall, this indicates that this liquid is silicone oil. Regarding the white crust, it is assumed to be precipitated surfactant (the same white deposit was obtained when drying samples of aqueous surfactant solution). In addition, we took a sample of the transparent phase $(\approx 1 \mathrm{~g})$ and diluted it in distilled water ( $\approx 15 \mathrm{~g})$. Gentle stirring with a spatula allows us to redisperse the transparent phase and gives a white opaque aqueous solution, similar to an $\mathrm{O} / \mathrm{W}$ emulsion. This means that the oil transparent phase is still in the form of small size droplets (see below).

These observations suggest that we missed something when examining the water and oil profiles obtained by MRI. First note that we do not detect by MRI a clear increase of the profile level in the region where the supernatant oil was observed. This may due to uncertainties and to the fact that the impact of replacing water by oil in some emulsion layer leads to a maximum increase of the oil signal by a factor 1.1, which is not easily observable in a logarithmic representation. A clearer observation in the MRI profile evolution is that of the maximum heights (i.e. thicknesses) of the oil and water profiles, which do not seem to vary in the same way during drying. For example (see 
Figure 4 and Figure 5) in the last stage of our tests, the height of the water profile goes on decreasing while the oil profile thickness remains constant. In order to quantify this phenomenon, we first remark that in logarithmic scale the profiles drop to zero around some point at the sample top, which takes the form of an almost vertical profile shape in this region. Then we estimate the profile thickness from the position of this drop, by arbitrarily taking the profile height for a volume/unit length of $0.2 \mathrm{~cm}^{-2}$, which is clearly in the almost vertical zone of the profile so that the error on the effective thickness is very small. We thus determine the thicknesses of the oil and water profiles in time. The difference between the former and the latter, which a priori represents a layer of pure oil above the emulsion, appears to increase as drying progresses. It is remarkable that this variation is linear with the time (see Figure 9), while the drying rate varies approximately with the thickness difference at a power -2 .

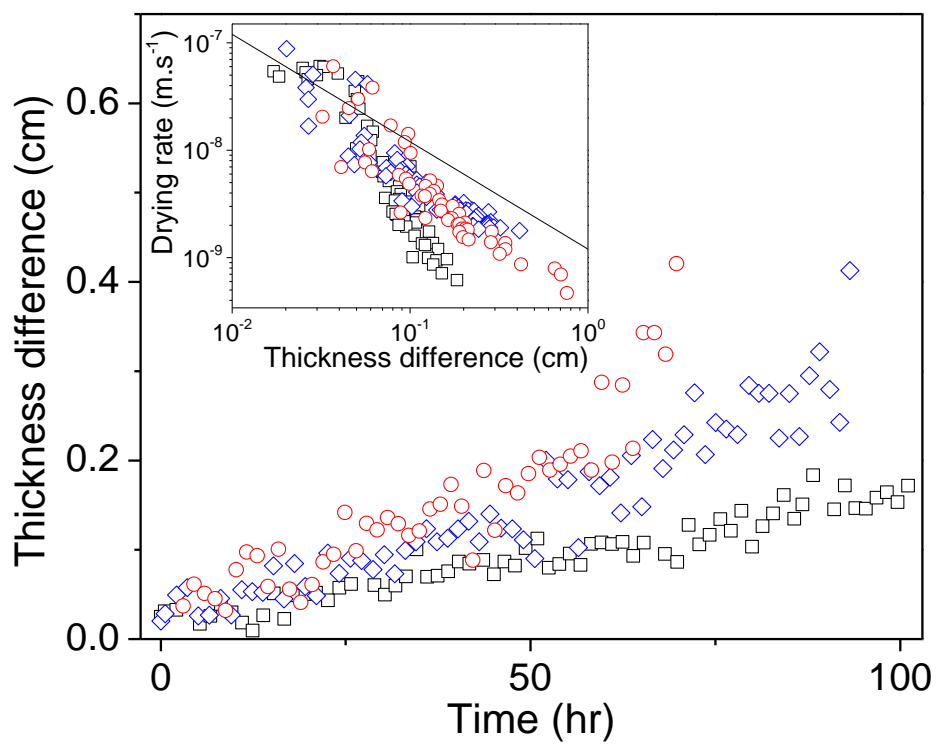

Figure 9 : Difference of oil and water profile thicknesses (see text) as a function of time for the experiments of drying of emulsions composed of $61 \%$ (black squares) and $81 \%$ (red circles) dried with an airflow of $0.1 \mathrm{~m} . \mathrm{s}^{-1}$ and $81 \%$ (blue diamonds) silicone oil dried with an airflow of $0.3 \mathrm{~m} . \mathrm{s}^{-1}$. The inset show the same data as a function of the drying rate. The line corresponds to drying associated to water diffusing through the (observed) oil layer according to theory.

\section{Discussion}

From these observations, we can infer the following mechanisms of emulsion drying. Starting from the lowest concentration for which we have a homogeneous emulsion (i.e. without sedimentation), the droplets first draw nearer to each other without being significantly deformed up to an oil concentration of about $70 \%$. During this stage the drying rate does not vary much, as water can easily move towards the free surface by rearranging the geometrical droplet configuration. Beyond $70 \%$ the droplets start to deform as the concentration is further increased, which induces large capillary stresses inside the material. Since evaporation first extracts water around the sample free surface, this process induces a disequilibrium between the capillary pressure of the liquid at the top and the bottom of the sample; inducing both a liquid transport throughout the sample, and a more or less progressive variation of the water concentration in the sample. This phenomenon may be described within the frame of poromechanics [35], which relies on the same physical principles developed by 
[26] but by dealing directly with macroscopic variables instead of at the local scale. The similarity of the shape of the profiles for water fraction below $20 \%$ suggests that there is some similarity of the physical processes at work, i.e. the suction force is increased in proportion to the resistance to water transport through the structure. Incidentally, it is worth noticing that even if some concentration gradient develops this does not significantly affect the mechanical properties of the sample, which are still close to those of an emulsion directly prepared at the same mean concentration. The origin of this phenomenon is the fact that the elastic modulus of an emulsion does not diverge when the concentration tends towards higher concentrations, so that the different regions of different concentrations roughly play a similar role. A different result would be obtained with a solid particle suspension for which the viscosity or elastic modulus would tend to infinity when the concentration tends to the maximum packing fraction.

Eventually, this water concentration gradient finishes by a catastrophic increase of the concentration around the free surface, which leads to coalescence. Thus a (pure) oil layer forms at the free surface that grows in thickness as drying proceeds, and which in turns induces a decrease of the drying rate in time. At first sight, it seems reasonable to think that this is the main process governing the decrease of the drying rate, especially when one sees that the drying rate decreases as the thickness of the (pure) oil layer increases (see Figure 9). The expected process in that case is a diffusion of liquid water molecules through the oil layer. A basic approach from Fick's law predicts a rate of diffusion, and consequently a drying rate, inversely proportional to the oil layer thickness. In order to check this we let dry a water layer, covered by a layer of pure silicone oil in a Petri dish, similarly to [36]. We found a drying rate of $3 \times 10^{-9} \mathrm{~m} . \mathrm{s}^{-1}$ for an oil layer of $4 \mathrm{~mm}$. Using this value we deduce the expected drying rate at any time as a function of the current thickness of the oil layer. It appears that this prediction is in good agreement during a first stage (see inset of Figure 9), say for an oil thickness smaller than $1 \mathrm{~mm}$. However, we start to have a significant discrepancy when the oil thickness increases further: the experimental drying rate is now 2 or 3 times smaller than expected from this model (see inset of Figure 9). This suggests that there is an additional effect contributing to slow down even more the drying process. Note that, in any event, we have no clear explanation for the origin of the variation of the oil thickness in time. Indeed, it can be expected to result from a complex process around the sample top, where the concentration gradient diverges, but we ignore the critical conditions for which coalescence will occur. Finally, it is remarkable, but also intriguing, that the oil layer thickness essentially increases linearly in time, as if the complex effects above mentioned would ultimately give a simple result.

We can suggest that the white crust also plays a significant role in the decrease of the drying rate at least in the second stage (i.e. oil layer thickness larger than about $1 \mathrm{~mm}$ ). It might behave as an additional porous medium (with very small pore size) that liquid water would have to cross before reaching the next step, i.e. diffusing through the oil layer. This layer most likely forms due to accumulation of surfactant molecules transported by the water beneath the oil layer. We verified this by calculating the Peclet number, which evaluates the relative contributions of water evaporation and surfactant diffusion to the distribution of the surfactant along the vertical axis. With an initial drying rate of $10^{-7} \mathrm{~m} \cdot \mathrm{s}^{-1}$ and emulsion thickness of $1.5 \mathrm{~cm}$, we obtain $P e=v H / D \approx 15$ with $D=10^{-10} \mathrm{~m}^{2} \cdot \mathrm{s}^{-1}$ the diffusion coefficient of the surfactant. $P e>1$ indicates that the surfactant is indeed likely to accumulate. When the surfactant concentration reaches the maximum solubility $\left(10 \% \mathrm{~m} / \mathrm{v}\right.$, which is equivalent to a mass fraction of $\left.9.1 \%_{\mathrm{wt}}\right)$ the surfactant precipitates. As more and more surfactant accumulates and precipitates, a crust forms. The higher the initial surfactant 
concentration is, the earlier the crust will form and the thicker it will be, since there is more surfactant available in the solution and therefore the drying rate will decrease earlier and faster. Such observations were made for the drying of porous media initially saturated with aqueous surfactant solutions (see Appendix 6)

Additional tests further confirm this analysis: i) varying the velocity of the air flux between 1.6 and $5.4 \mathrm{~m} . \mathrm{s}^{-1}$ the initial velocity in the very first time varied slightly, but we did not observe significant variation of the rest of the drying rate curve, suggesting no significant impact of the air flux, at least in this range; also the effect on the concentration distribution was observed (by MRI) to be minimal, with very similar evolutions of the profiles for two different air fluxes $(0.1$ and $0.3 \mathrm{~m} / \mathrm{s})$ at a concentration of $80 \%$; ii) for a given initial water fraction the drying rate decreases less rapidly when the sample thickness or the surfactant concentration is smaller (see Figure 10), which both result in a smaller amount of surfactant associated with some elementary drying of the emulsion sample. These observations are consistent with the above description of the process: the oil layer forms and tends to control drying during a first stage, growing and decreasing the drying rate (which soon becomes independent of external air flux velocity), while the accumulation of surfactants further contributes to decrease the drying rate.

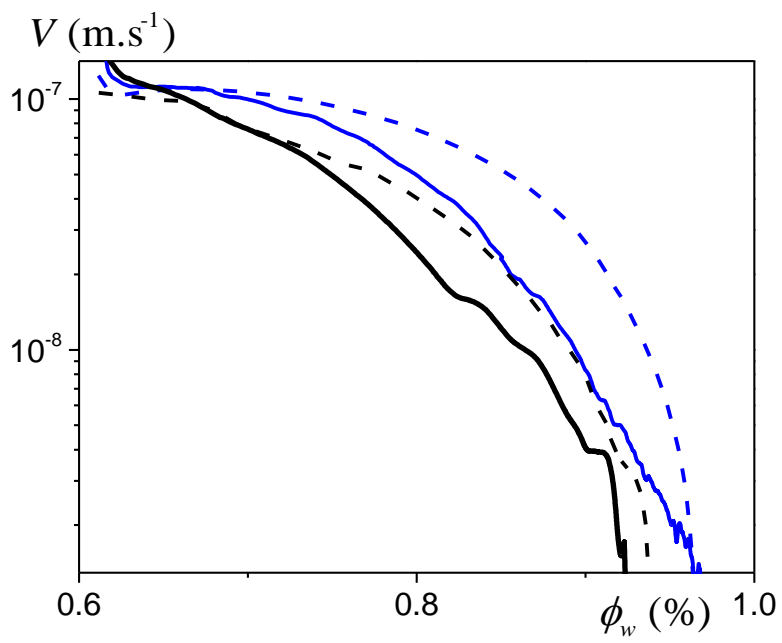

Figure 10 : Drying rate as a function of oil fraction for $1.5 \mathrm{~cm}$ (continuous lines) or $0.6 \mathrm{~cm}$ (dashed lines) thick (initially) $61 \%$ emulsion with $3 \%_{\text {wt }}$ (black) or $1.14 \%_{\text {wt }}$ (blue) TTAB. The red line corresponds to an initially $81 \%$ emulsion with $3 \%_{\mathrm{wt}}$ TTAB.

Finally one of the most striking observation is that such a thick layer of emulsion shrinks almost homogeneously during drying, with some slight gradient of concentration from the free surface to the bottom. A full modelling within the frame or poromechanics with a detailed comparison with data can certainly be attempted, but it has to take into account the large variations of the drying rate in time or even propose some coupling between this rate and the history of drying, a rather difficult task. Here we will simply rely on some predictions of the model developed by Sprakel's group [26] to discuss further the consequences of our observations. This model describes the evolution of the local emulsion concentration when some interstitial liquid is extracted from one boundary, leading to a compression which propagates through the sample. The dimensionless equations of this model describe the evolution of the local porosity (i.e. the water fraction) as a function of the distance rescaled by the droplet size, the time rescaled by the viscosity times the droplet size divided by the surface tension. In Figure 11 we show the results of such modelling under a given rate of extraction 
(i.e. drying rate) for three different layer thicknesses, for an initial (uniform) porosity of 0.36 . We see that similar boundary conditions induce a stronger gradient throughout the material for an increasing sample thickness: for a small initial sample thickness the porosity distribution remains almost uniform (though necessarily a slight gradient exists) down to low porosity; for a large sample thickness the extraction of liquid essentially occurs close to the free surface giving rise to a strong gradient at the approach of the free surface while the rest of the material remains unaffected. Thus we deduce that under similar boundary conditions, if we neglect the impact of the drying rate evolution, the porosity distribution will be more homogeneous as the initial sample thickness decreases. Under these conditions we deduce that for our emulsions, thinner layers would dry even more homogeneously.
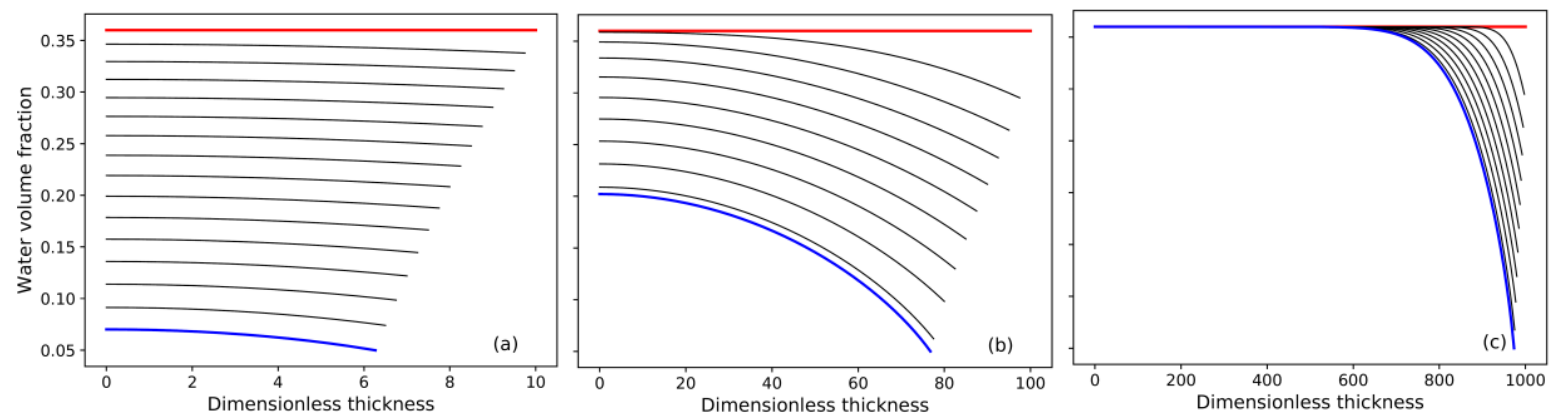

Figure 11 : Successive porosity distribution in time during drying of an emulsion layer for a fixed drying rate for different initial dimensionless thicknesses ((a) 10, (b) 100, (c) 1000), according to a poromechanical model [26]. Note that the time interval between two successive profiles is increased for increasing thicknesses.

On the other side, considering that the drying rate mainly varies with the thickness of the oil layer and the surfactant accumulated around the top free surface, the drying rate will be less affected for smaller initial emulsion layer. Indeed, since the amount of oil and accumulated surfactants are a priori proportional to the sample volume, to reach a given (mean) oil concentration, a thinner emulsion layer will yield thinner layers of oil and surfactants, leading to a lower decrease of the drying rate with regards to the initial one.

\section{CONCLUSION}

This experimental investigation led us to present the basic properties of drying of thick emulsion layers. As water is extracted from the sample free surface, the emulsion shrinks almost homogeneously, and its structure and strength also vary essentially homogeneously. The application of a poromechanics model allows to deduce that, under similar drying rate, thinner layers of emulsions would dry even more homogeneously.

On the other side, the drying rate decreases continuously as water is extracted from the sample, in contrast with many porous systems for which a long regime at constant drying rate is first observed. It appears that even for such thick layers, local effects close to the free surface govern the drying 
rate: coalescence of some oil droplets just below the free surface, and surfactant accumulation just below.

This implies that the full description of this process through a model, able to predict the evolution of the drying rate and the porosity distribution, appears rather complex. It should obviously be based on poromechanics concepts, but should also consider the coalescence of oil droplets resulting from the concentration gradient and the surfactant transport and their impact on the drying rate.

\section{Appendix 1: Magnetic Resonance Imaging}

In order to get one-dimensional profiles of the content of the various fluids along the vertical direction, three relaxation-weighted $1 \mathrm{D}$ imaging sequences were used (see Figure A1) denoted IR, SR and CPMG respectively in the following. The sequence IR is based on an inversion-recovery module and is repeated for various values of the $\tau$ delays to probe the kinetics of spin-lattice relaxation. The sequence SR performs the same, but uses instead a saturation-recovery module. The last sequence relies on the CPMG sequence [37], [38] and probes spin-spin relaxation. For signal detection and 1D profiling, all sequences are terminated with a double spin-echo imaging module, which allows the correction of measurement bias due to spin-spin relaxation and field inhomogeneities during the imaging period [39]. The sample holder is not detected in our experiments due to its very short spinspin relaxation time regarding the echo time TE.

a) IR

Echo 1 Echo 2

b) SR
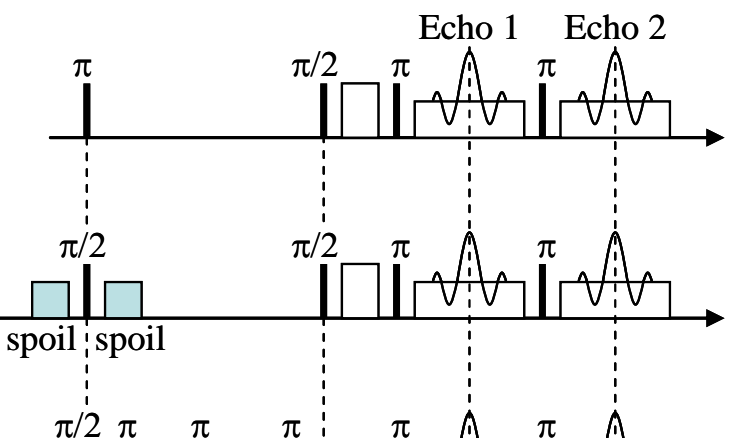

c) CPMG

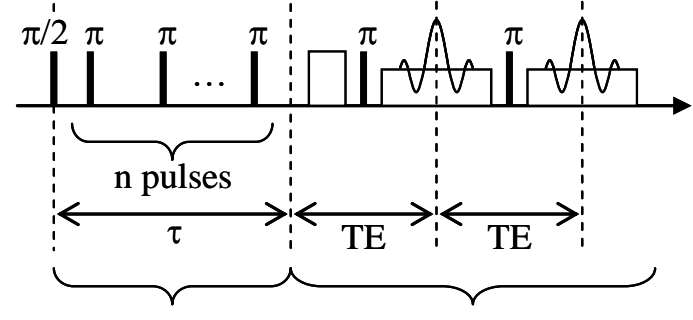

Relaxation

1D Imaging

Figure A1 : Three relaxation-weighted MRI sequences used for the separate detection of water and oil in the drying emulsion, based on the inversion-recovery (a), saturation-recovery (b), and CPMG (c). White rectangles represent the imaging gradient pulses applied in the vertical direction.

In the specific case of the IR sequence, the raw MRI signal obtained for a given $\tau$ parameter at some given vertical coordinate $z$ was modeled as:

$M(\tau, m) \approx\left(A-2 M_{W} \exp \left(-\tau / T_{1}\right)\right) \exp \left(-m R_{W} T E\right)+M_{o} f_{O}(\tau) \exp \left(-m R_{O} T E\right)$ 
Where $m=1,2$ refers to the first or second imaging echo, and $A, M_{W}, T_{1}, R_{W}, M_{O}$ and $R_{O}$ are fitting parameters, obtained at a given $z$ position by means of a simultaneous least square fit of all signals obtained for all values of $\tau$ and $m$. The first part of the RHS describes the monoexponential relaxation of the water phase with a-local- equilibrium magnetization $M_{W}$ and a characteristic time $T_{1}$. This was found to be a reasonable assumption due to the high probability of a fast-exchange relaxation regime of water between oil droplets (Brownstein). $T_{1}$ is left as a free parameter because it is highly sensitive to the ratio between the external area of the droplets and the water amount, and is then likely to evolve when water evaporates and droplets concentrate (see Figure A2). The second part describes the relaxation of the oil phase, the pure phase of which was found not to follow a mono-exponential behavior. Since on the contrary, its relaxation kinetics is quite independent of droplet size and water concentration (see Figure A2), it was described by means of a fixed normalized abacus $f_{O}(\tau)$ experimentally measured on a sample of pure oil, and only the associated signal intensity $M_{O}$ was left as a fitting parameter. Extreme accuracy was required on $f_{o}(\tau)$ to make the processing work. Since small fluctuations in either temperature or hardware behaviour could affect $f_{O}(\tau)$, sample of pure silicone oil ( $\left.\approx 100 \mathrm{~g}\right)$ contained in a glass Petri dish 10 $\mathrm{cm}$ wide was placed beneath the emulsion sample in the MRI magnet so as to get constantly updated measurements of $f_{O}(\tau)$ at each step of the experiment. $R_{W}$ and $R_{O}$ finally take into account the coupled relaxation/spin defocusing effects during the imaging module, regarded as different for water and oil. $M_{W}$ and $M_{O}$ are physically proportional to the local water and oil mass respectively, and converted into a local oil and water content in the emulsion by means of a proper calibration. A similar modelling and treatment (not detailed here) was applied to SR and CPMG sequences.

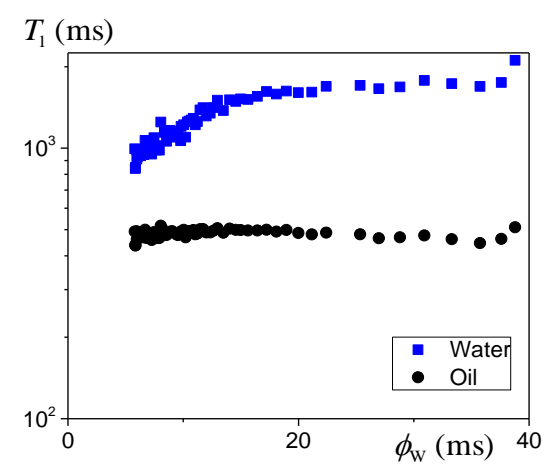

Figure A2 : Evolution of average longitudinal relaxation time $T_{1}$ of $O / W$ silicone oil emulsion during drying as a function of water volume fraction. $T_{1}$ of water (blue squares) decreases significantly during the drying, while it remains constant for oil (black circles).

For spin-lattice as well as for spin-spin relaxation, the ratio of the average relaxation times of water and oil was hardly higher that 3 or 4 , which is prone to compromise the fitting stability, and thus the actual ability to accurately separate water and oil. Some specific attention was brought here by using 4 (for IR and SR sequences) or 3 (for CPMG) values of $\tau$ optimized numerically so as to minimize the sensitivity of fitted parameters on measurement noise. The list of $\tau$ delays was updated at some moment in the experiments in order to follow the evolution of sample properties. Additionally the $T_{1}$ parameter during the fitting procedure was constrained in a fixed research interval $T_{L}<T_{1}<T_{U}$, 
where $T_{L}$ tried to fix a limit between what should be attributed to water or oil regarding relaxation kinetics. When too much sensitivity to the exact value of $T_{L}$ was found, it was concluded that the relaxation behavior of the two phases became so close that a proper phase separation was not possible anymore. This was usually the case at the very end of experiments, which should then be considered as blind (see Figure A3).

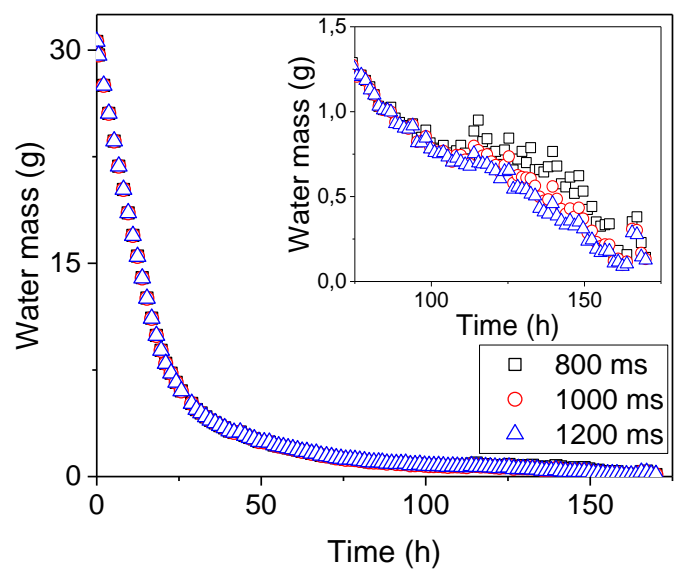

Figure A3 : Evolution of the total measured mass of water of an O/W emulsion with an initial oil volume fraction $61 \%$ during drying obtained through $T_{1}$ weighted profiles (IR) using three different lower bounds for the $T_{1}$ parameter. Inset shows close up of the final hours of drying. Past some delay of about $100 \mathrm{~h}$, the measurements become highly sensitive to the bound, indicating that MRI is no more able to well distinguish water and oil.

Due to the various issues owing to the validity of the mixed exponential / abacus relaxation model of the sample, the necessary real-time update of the oil behavior, and the fitting stability [40], the three MRI sequences were repeated in cycles lasting approximately one hour and a half to cross-validate the measurements during the drying of the sample. Except for the very last times of the drying as explained above, all sequences gave very similar results (see Figure A4), which thus confirmed the robustness of the measurements. We only presented here the - validated - results pertaining to the IR sequence.

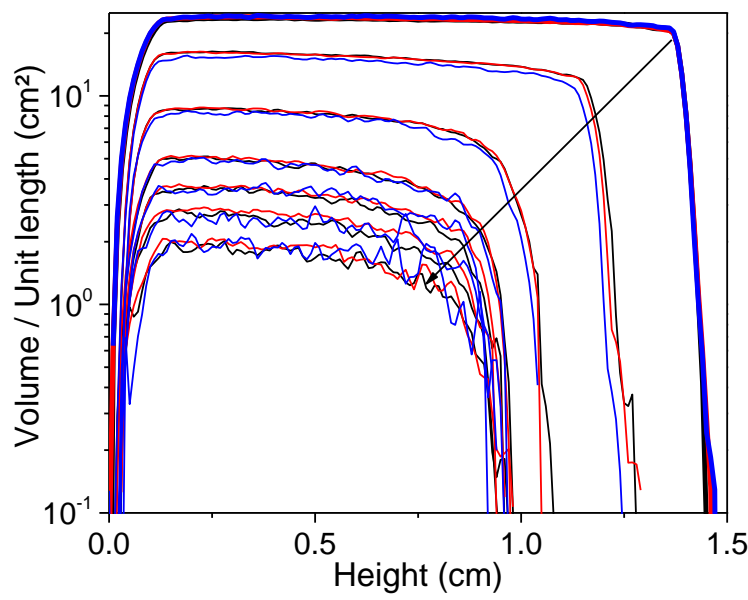

Figure A4 : Water distribution profiles of a $61 \%$ silicone emulsion during drying obtained after 0 , $10,20,30,40,60$ and 70 hours of drying approximately. Drying follows the direction of the 
arrow. Measurements obtained from IR, SR and CPMG sequences are represented in black, red and blue respectively. Initial profiles before drying has begun are represented in thick lines.

As an additional control of the MRI measurements, the sample was taken out of the MRI to be weighted between some of the sequence cycles. As during this operation the sample was exposed to ambient air for less than 3 minutes at a time while some significant drying (say, 5\% of the initial water mass) requires at least two hours, this external weighing had no effect on the drying process. The total water content measured with MRI and that deduced from weighing always evolved as parallel curves (see Figure A5) for a given sample. However, a systematic difference was found of about $1 \mathrm{~g}$, which for a given sample remained constant from the beginning to the end of the drying. We checked, by drying and weighing the final sample, that there effectively remains a water amount larger than estimated by MRI, of about $1 \mathrm{~g}$. In other words, not all the water can be observed by MRI measurements.

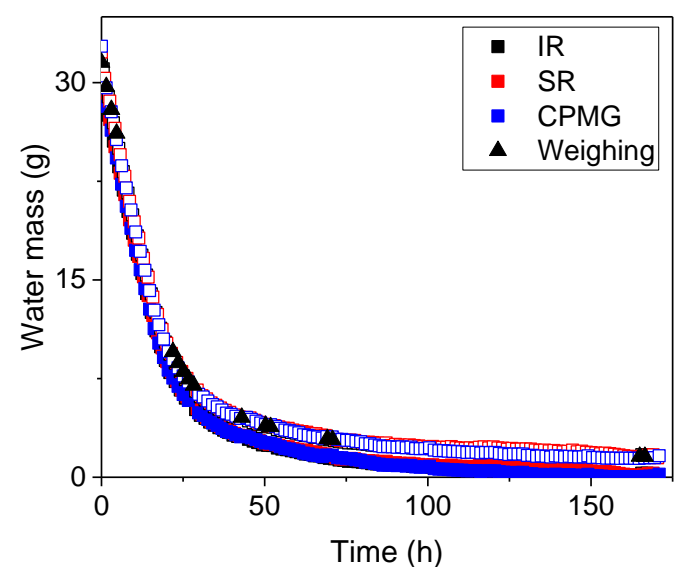

Figure A5 : Evolution of the total mass of water of a $61 \%$ silicone emulsion during drying obtained through weighing and MRI measurements (through different techniques, see text). MRI measurement results are represented with filled squares, and weighing results with filled triangles. Empty squares represent MRI results to which $1.4 \mathrm{~g}$ was added. 


\section{Appendix 2: Elastic and loss modulus of an emulsion as a function of the strain amplitude}

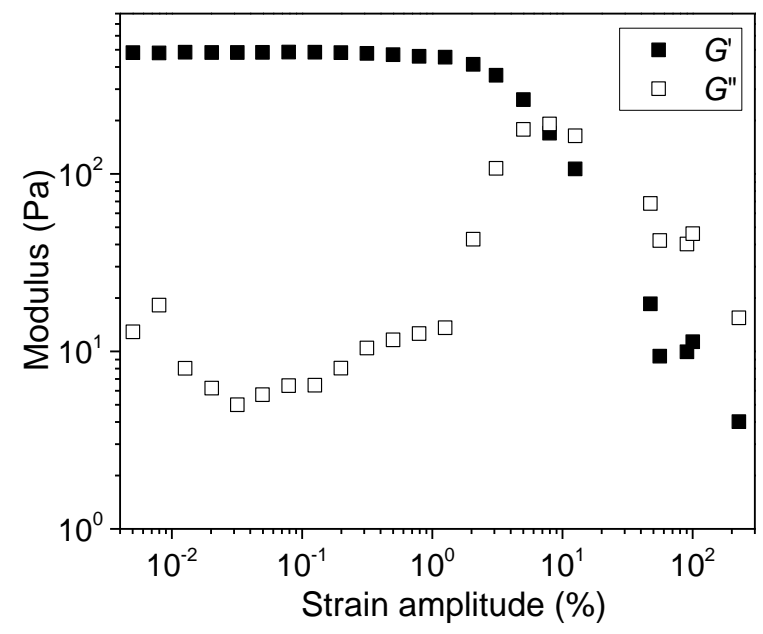

Figure A6 : Elastic and viscous moduli $\mathrm{G}^{\prime}$ and $\mathrm{G}^{\prime \prime}$ as a function of shear strain $\gamma$ of an O/W silicone emulsion with an $81 \%$ oil volume fraction.

\section{Appendix 3. Concentration of free surfactants.}

TTAB is a quaternary ammonium salt used to stabilize direct silicone emulsions. Part of the surfactant adsorbs at the oil/water interfaces, while the rest of surfactant molecules form micelles in the aqueous phase. Depending on the surfactant concentration in the aqueous phase, as well as the temperature, the micelle arrangement varies, from spherical to long rod-shaped arrangements [41], and forms different phases [42]. Here we estimate the surfactant concentration in the aqueous phase that has not adsorbed at oil/water interfaces. We assume oil droplets are spherical and monodisperse, with a radius $R=3 \mu \mathrm{m}$, and that no coalescence occurs during drying. We consider that the surface of an oil droplet is covered with surfactant molecules, and that the space occupied by each surfactant molecule is equal to $\pi r^{2}$ (with $r=2.6 \dot{A}=2.6 .10^{-10} \mathrm{~m}$ [43]). We deduce that the maximum number of surfactant molecules covering the surface of one droplet is $N \approx 4 \pi R^{2} / \pi r^{2}$. The number of oil droplets is $N_{0}=\left(\phi \Omega_{w} / 1-\phi\right) / 4 \pi R^{3} / 3$ (where $\Omega_{w}$ is the water volume), and we deduce the maximum mass of adsorbed surfactant molecules:

$m_{A}=N N_{0} \frac{M}{N_{a}}$

with $N_{a}$ the Avogadro number. If the mass fraction of surfactant is $\phi_{S}$ the total mass of surfactants is $m=\phi_{S} \rho \Omega /\left(1-\phi_{S}\right)$. We can then calculate the mass of free TTAB in the aqueous phase $m_{F}=m-$ $m_{A}$ and the mass fraction of free TTAB: $\phi_{F}=m_{F} /\left(m_{F}+\rho \Omega\right)$.

The emulsions we dried have an initial oil volume fraction of 61,71 and $81 \%$ with $\phi_{S}=3 \%$. For these three emulsions $\phi_{F}$ is found to be respectively $2.6 \%, 2.4 \%$ and $1.9 \%$. At the end of the different drying experiments (for the specific tests of Figure 3 in which the test is stopped before the end of drying), it is equal to $7.2 \%, 10.3 \%$ and $4.2 \%$. 
According to [42], the surfactant molecules begin to arrange themselves in rod-like micelles, forming a viscous phase starting at $40 \%\left(\mathrm{~T}=22^{\circ} \mathrm{C}\right)$. Therefore, at the surfactant concentrations observed here during drying, the surfactant micelles should not form a viscous phase during the drying process, the solution viscosity is expected to be rather close to the solvent viscosity. It therefore seems unlikely that the micelles are solely responsible for the observed increase of the viscous moduli during drying.

\section{Appendix 4: Comparison of the gradients in MRI profiles for two different initial concentrations}

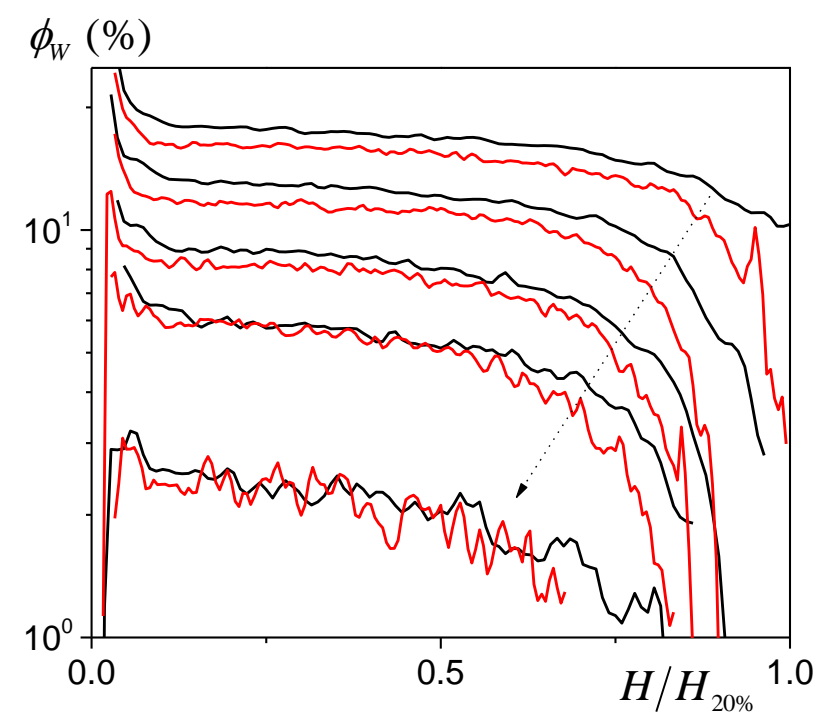

Figure A7 : Drying of two O/W emulsions initially composed of $61 \%$ (in black) and $81 \%$ (in red) silicone oil and containing $1.14 \%_{\mathrm{wt}}$ and $3 \%_{\mathrm{wt}}$ surfactant in aqueous phase respectively: water concentration profiles for different water contents (from top to bottom, 16-12-8-5-2\%) as a function of height normalized by $H_{20 \%}$ (1.62 and $1.80 \mathrm{~cm}$ respectively). The arrow indicates the evolution in time.

\section{Appendix 5: Oil droplet size evolution}

Confocal microscopy images of a drying fluorescent emulsion show that at the initial oil volume fraction of $61 \%$, the oil droplets appear to be only marginally deformed (see Figure A8). Indeed the oil volume fraction is below $\phi_{c} \approx 0.64$ the critical value for random close packing of solid spheres. In our case, since the emulsion is slightly polydisperse with oil droplet radius ranging between 2 and $5 \mu \mathrm{m}$ (see Figure A9), the critical value for random close packing is most likely a little higher. 


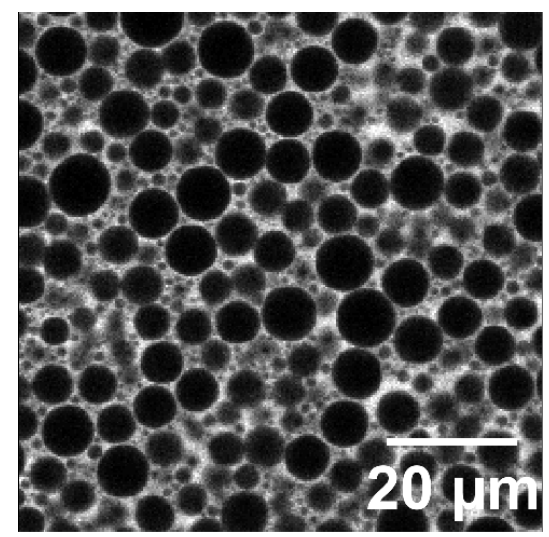

(a)

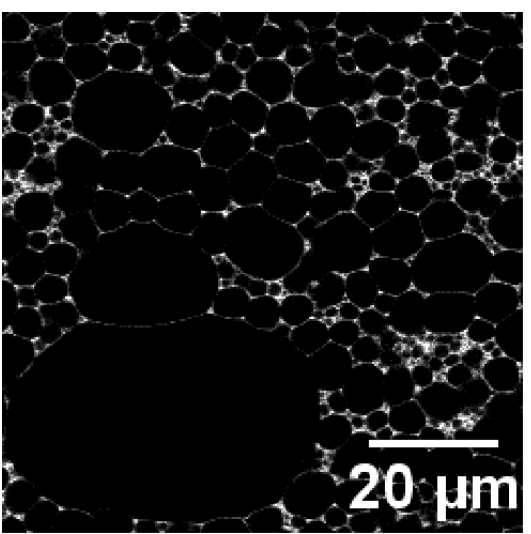

(d)

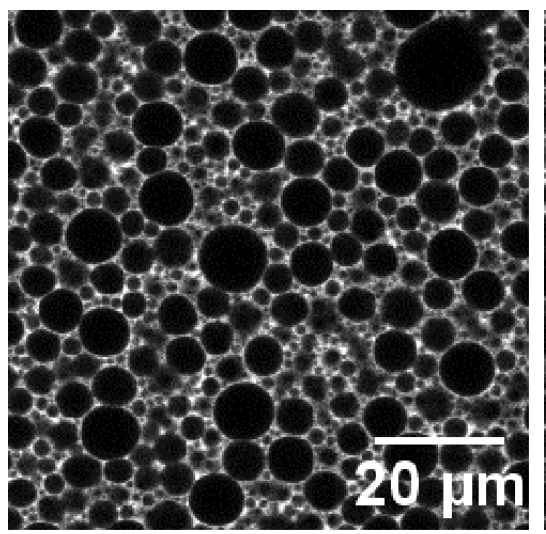

(b)

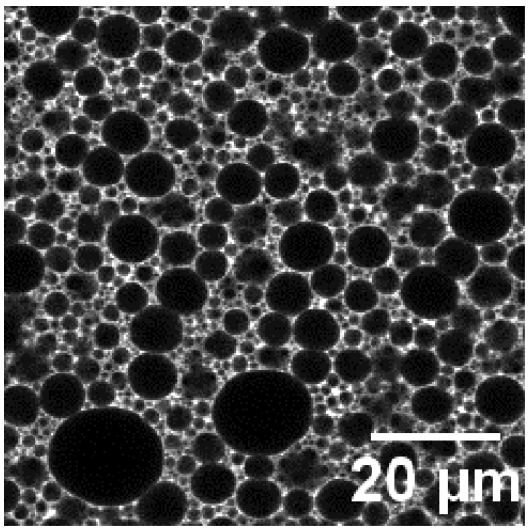

(c)

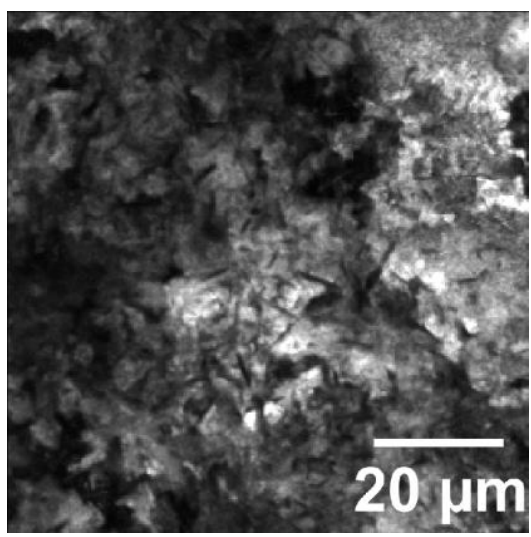

(e)

Figure A8 : Confocal microscope images of an O/W emulsion with an initial oil volume fraction of $61 \%$ and containing $3 \%_{\mathrm{wt}}$ surfactant in aqueous phase at different stages. The oil droplets appear in black, while water appears in white. (a) Initial state $\phi=61 \%$; (b) $\phi=70 \%$ (c) $\phi=80 \%$; (d) transparent phase and (e) white crust $\phi=95 \%$.

As drying progresses, the oil droplets come closer together and eventually deform. When very little water is left in the emulsion (less than $5 \%$ ), the droplets are very closely packed (see Figure A8d). Although bigger and bigger oil droplets can be found in the emulsion, the oil droplet size distribution does not evolve significantly throughout drying indicating that coalescence is limited in the bulk of the emulsion (see Figure A9). 


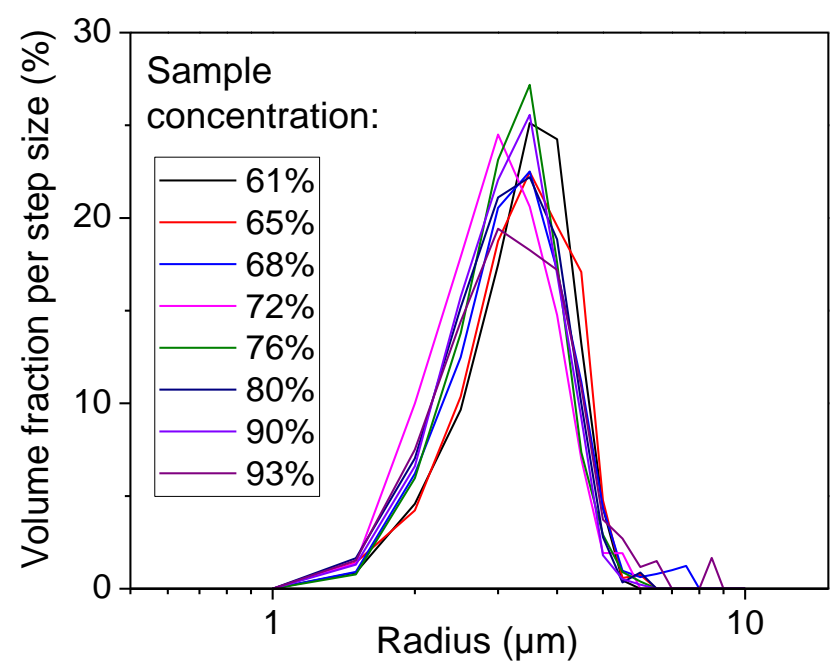

Figure A9 : Evolution of droplet size distribution of drying O/W emulsion with an initial oil volume fraction of $61 \%$.

The thin white crust at the sample free surface has a completely different aspect (see Figure A8e): the sample is non-uniform with some very opaque areas (in black and dark gray) and more translucent areas (in white). We can suppose that this is due to the varying thickness of the white crust, which we had observed to be non-uniform.

A sample of the supernatant oil layer was also observed but nothing was visible with the confocal microscope. As fluorescein is water-soluble but not oil-soluble, it would appear that the fluid is indeed pure oil.

Appendix 6: Effect of surfactant on the drying rate of a granular packing.

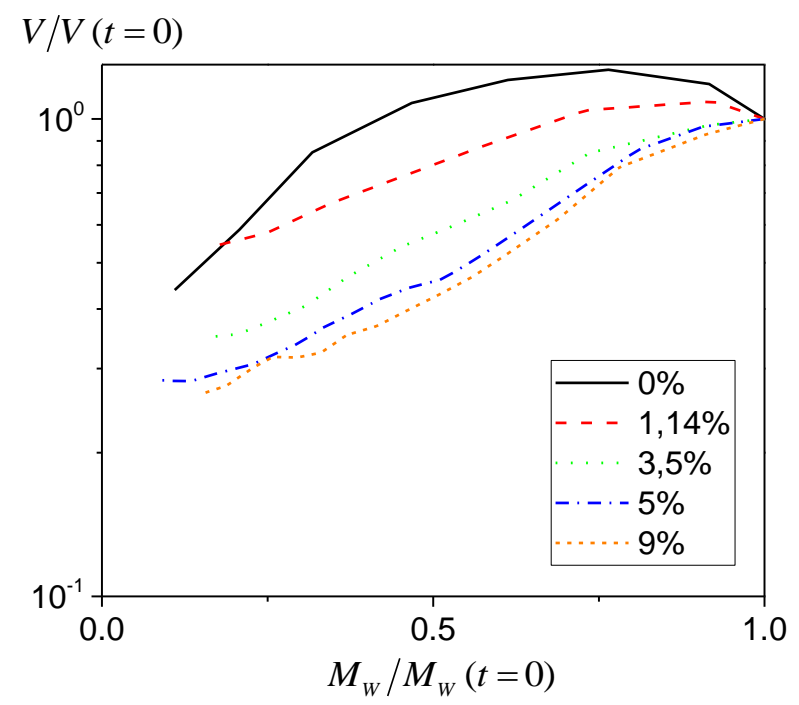

Figure A10 : Drying curves obtained for the drying of a bead packing ( $\varnothing=315-500 \mu \mathrm{m}$ ) initially saturated with aqueous surfactant solutions. The initial surfactant concentration is indicated on the graph.

We dried bead packings used as model porous media initially saturated with aqueous surfactant solutions ( $\left.[T T A B]=0-9 \%_{w t}\right)$ under a dry airflow and found that the higher the initial concentration of the surfactant was the more the drying rate decreased (see Figure A10). For concentrations higher 
than $5 \%_{w t}$, the constant rate period generally observed for the drying of porous media at high water saturation levels is non-existent. We can assume that in such cases, the surfactant precipitates almost immediately and limits water flow through the porous media.

\section{References}

[1] B. P. Binks, P. D. I. Fletcher, B. L Holt, P. Beaussoubre, K. Wong, Langmuir, 2010, 26, 1802418030

[2] B. P. Binks, J. Brown, P.D.I. Fletcher, A.J. Johnson, I. Marinopoulos, J.M. Crowther, M.A. Thompson, ACS Appl. Mater. Interfaces, 2016, 8, 13270-13281

[3] P. K. Weissenborn, A. Motiejauskaite, J. Coat. Technol., 2000, 72, 65-74

[4] H. M. van der Kooij, J. Sprakel, Soft Matter, 2015, 11, 6353-6359

[5] D. Lesueur, C. Coupé, M. Ezzarougui, Road Mater. Pavement Design, 2001, 2, 161-179.

[6] A. Gharsallaoui, G. Roudaut, O. Chambin, A. Voilley, R. Saurel, Food Res. Int., 2007, 40, 11071121

[7] J. H. Chew, N. Fu, T. Gengenbach, X. D. Chen, C. Selomulya, J. Food Eng., 2015, 157, 33-40

[8] T. G. Mason, J. Bibette, D. A. Weitz, Phys. Rev. Lett., 1995, 75, 2051-2054

[9] T. G. Mason, J. Bibette, D. A. Weitz, J. Colloid Interface Sci., 1996, 179, 439-448

[10] J. Van Brakel, " Mass Transfer in Convective Drying ", in Advances in Drying, vol. 1, Hemisphere Publishing Corporation, 1980, 217-267

[11] M. Prat, Int. J. Multiph. Flow, 1993, 19, 691-704

[12] P. Coussot, Eur. Phys. J. B, 2000, 15, 557-566

[13] I. N. Tsimpanogiannis, Y. C. Yortsos, S. Poulou, N. Kanellopoulos, A. K. Stubos, Phys. Rev. E, $1999,59,4353-4365$

[14] P. Lehmann, S. Assouline, D. Or, Phys. Rev. E, 2008, 77, 056309

[15] N. Shokri, P. Lehmann, D. Or, Water Resour. Res., 2009, 45, W02415

[16] T. M. Shaw, Phys. Rev. Lett., 1987, 59, 1671-1674

[17] A. G. Yiotis, D. Salin, E. S. Tajer, Y. C.ortsos, Phys. Rev. E, 2012, 86, 026310

[18] J. Thiery, S. Rodts, D. A. Weitz, P. Coussot, Phys. Rev. Fluids, 2017, 2, 074201

[19] A. Kapilashrami, K. Eskilsson, L. Bergström, M. Malmsten, Colloids Surf. Physicochem. Eng. Asp., 2004, 233, 155-161 
[20] E. Keita, T. E. Kodger, P. Faure, S. Rodts, D.A. Weitz, P. Coussot, Phys. Rev. E , 2016, 94, 033104

[21] J.-P. Gorce, D. Bovey, P. J. McDonald, P. Palasz, D. Taylor, J. L. Keddie, Eur. Phys. J. E, 2002, 8, 421-429

[22] D. Guigner, C. Fischer, Y. Holl, Langmuir, 2001, 17, 3598-3606

[23] D. Guigner, C. Fischer, Y. Holl, Langmuir, 2001, 17, 6419-6425

[24] D. Guigner, C. Fischer, Y. Holl, Polym. Int., 2003, 52, 448-456

[25] H. Feng, J. Sprakel, D. Ershov, T. Krebs, M. A. C. Stuart, J. van der Gucht, Soft Matter, 2013, 9, $2810-2815$

[26] H. Feng, J. Sprakel, J. van der Gucht, Phys. Rev. E, 2015, 92, 023011

[27] K. Hasegawa, S. Inasawa, Soft Matter, 2017, 39, 7026-7033

[28] E. Ciampi, U. Goerke, J. L. Keddie, P. J. McDonald, Langmuir, 2000, 16, 1057-1065

[29] J. D. Griffith, A. E. Bayly, M. L. Johns, Chem. Eng. Sci., 2008, 63, 3449-3456

[30] J. D. Griffith, J. Mitchell, A. E. Bayly, M. L. Johns, J. Mater. Sci., 2009, 44, 4587-4592

[31] H. M. van der Kooij, M. de Kool, J. van der Gucht, J. Sprakel, Langmuir, 2015, 31, 4419-4428

[32] G. Ovarlez, S. Rodts, A. Ragouillaux, P. Coussot, J. Goyon, A. Colin, Phys. Rev. E, 2008, 78036307

[33] P. Coussot, Rheometry of Pastes, Suspensions, and Granular Materials: Applications in Industry and Environment. John Wiley \& Sons, New York, 2005

[34] J. Thiery, E. Keita, S. Rodts, D. Courtier Murias, T. Kodger, A. Pegoraro, P. Coussot, Eur. Phys. J. $E, 2016,39,117$

[35] O. Coussy, Mechanics and Physics of Porous Solids. John Wiley \& Sons, New York, 2011

[36] H. Miyazaki, S. Inasawa, Soft Matter, 2017, 13, 8990-8990

[37] H. Y. Carr, E. M. Purcell, Phys. Rev., 1954, 94, 630-638

[38] S. Meiboom, D. Gill, Rev. Sci. Instrum., 1958, 29, 688-691

[39] M.D. Seck, E. Keita, P. Faure, P. Cavalié, M. Van Landeghem, S. Rodts, P. Coussot, Chem. Eng. Sci., 2016, 148, 203-211

[40] R.J.S. Brown, J. of Magn. Reson. 1969, 1989, 82, 539-561

[41] K. Holmberg, B. Jonsson, B. Kronberg, and B. Lindman, in Surfactants and Polymers in Aqueous Solution, Wiley-Blackwell, 2003, pp. 67-96.

[42] Ö. Masalci, M. Okcan, and N. Kazanci, J. Mol. Struct., vol. 843, 32-37 (2007)

[43] K. L. Mittal and P. Botherel, Eds., Surfactants in Solution: Volume 4. Springer US, 1986. 
